\title{
The Development of Consensus-Based Descriptors for Low-Acuity Emergency Medical Services Cases for the South African Setting
}

\author{
Faisal Binks, MBA; $\odot$ Lee Alan Wallis, $\mathrm{PhD}$; $\odot$ Willem Stassen, $\mathrm{PhD} \odot$
}

Division of Emergency Medicine, University of
Cape Town, Cape Town, South Africa
Correspondence:
Faisal Binks, MBA
Division of Emergency Medicine
University of Cape Town
Cape Town, South Africa
E-mail: faisal.binks@gmail.com

Conflicts of interest: The authors declared no conflicts of interest.

Keywords: emergency dispatch; Emergency Medical Service; low acuity; prehospital; triage

\author{
Abbreviations: \\ ED: emergency department \\ EMD: Emergency Medical Dispatch \\ EMS: Emergency Medical Service \\ LMIC: low- and middle-income countries \\ RTA: road traffic accident
}

Received: September 7, 2020

Revised: October 21, 2020

Accepted: November 3, 2020

\section{doi:10.1017/S1049023X21000169}

(C) The Author(s), 2021. Published by Cambridge University Press on behalf of the World Association for Disaster and Emergency Medicine. This is an Open Access article, distributed under the terms of the Creative Commons Attribution licence (http:// creativecommons.org/licenses/by/4.0/), which permits unrestricted re-use, distribution, and reproduction in any medium, provided the original work is properly cited.

\begin{abstract}
Introduction: Emergency Medical Services (EMS) are designed to respond to and manage patients experiencing life-threatening emergencies; however, not all emergency calls are necessarily emergent and of high acuity. Emergency responses to low-acuity patients affect not only EMS, but other areas of the health care system. However, definitions of low-acuity calls are vague and subjective; therefore, it was necessary to provide a clear description of the lowacuity patient in EMS.
\end{abstract}

Aim: The goal of this study was to develop descriptors for "low-acuity EMS patients" through expert consensus within the EMS environment.

Methods: A Modified Delphi survey was used to develop call-out categories and descriptors of low acuity through expert opinion of practitioners within EMS. Purposive, snowball sampling was used to recruit 60 participants, of which 29 completed all three rounds. An online survey tool was used and offered both binary and free-text options to participants. Consensus of $75 \%$ was accepted on the binary options while free text offered further proposals for consideration during the survey.

Results: On completion of round two, consensus was obtained on $45 \%$ (70/155) of the descriptors, and a further 30\% (46/155) consensus was obtained in round three. Experts felt that respiratory distress, unconsciousness, chest pain, and severe hemorrhage cannot be considered low acuity. For other emergency response categories, specific descriptors were offered to denote a case as low acuity.

Conclusion: Descriptors of low acuity in EMS are provided in both medical and trauma cases. These descriptors may not only assist in the reduction of unnecessary response and transport of patients, but also assist in identifying the most appropriate response of EMS resources to call-outs. Further development and validation are required of these descriptors in order to improve accuracy and effectiveness within the EMS dispatch environment.

Binks F, Wallis LA, Stassen W. The development of consensus-based descriptors for lowacuity Emergency Medical Services cases for the South African setting. Prehosp Disaster Med. 2021;36(3):287-294.

\section{Introduction}

Emergency Medical Services (EMS) are designed to respond to and manage patients experiencing life-threatening emergencies; however, not all responses by EMS are for emergent or life-threatening conditions. Emergency Medical Services have the potential to respond more efficiently to the higher acuity patient, which is hindered by the availability of vehicles and resources transporting the low-acuity patient. ${ }^{1}$ High volume response to non-emergency calls with lights and sirens not only increases the risk of motor vehicle accidents, ${ }^{2}$ but also predisposes EMS staff to factors such as poor staff morale, poor job satisfaction, skills decay, and fatigue., ${ }^{2,3}$

With regards to the emergency department (ED), there exists a substantial amount of research on the low-acuity (non-emergent) patient ${ }^{4,5}$ and the negative consequences these patients have on the higher acuity patients. Some of the research includes overcrowding and the time dedicated to the low-acuity patient in the ED. It has been shown that the arrival of one low-acuity patient to an ED significantly impacts the waiting times for treatment provided to a patient of high acuity. ${ }^{4} \mathrm{It}$ is also noted that one-third of $\mathrm{ED}$ patients are low acuity and a major contributor to this proportion is ambulance arrivals. ${ }^{6}$ One study has shown that $41 \%$ of patients transported to hospital were deemed to be low acuity and $79 \%$ of these patients could have been safely treated in a primary care center. ${ }^{5}$ 
Much research has been published around prehospital discharge or treat-and-refer procedures internationally. There is also indication that telephonic assessment of emergency calls identified patients that were less likely to require treatment in the ED and therefore had the ability to reduce unwarranted response of emergency vehicles to some calls. ${ }^{7} \mathrm{~A}$ further study showed that telephonic advice for these calls had proven to be safe and recommended full implementation in a clinical trial setting. ${ }^{8}$

In the US alone, it is estimated that US $\$ 560$ million could be saved annually through the appropriate management of patients with lower acuity. ${ }^{6}$ Consideration may be provided for emergency care practitioners in local EMS systems to provide on-scene discharge of patients and that the exact operational procedures for this requires further consideration and research prior to implementation. ${ }^{9}$ Although prehospital discharge is a useful mechanism in reducing low-acuity patient transport to hospitals, it does not alleviate the impact of the initial ambulance response to these calls. Dispatching of EMS resources timeously and appropriately based on patient acuity would be the ideal dispatching system, balancing patient safety and adequate resource allocation and utilization. ${ }^{8}$

In South Africa, the problem of lack in emergency resources is well-established with only 2,000 of the required 5,700 ambulances being in operation, ${ }^{10}$ therefore compounding the highlighted predisposing factors with emergent response to low-acuity calls. It was evidenced that $39 \%$ of patients presenting to an ED were transported by ambulance, ${ }^{11}$ and $47 \%$ of these patients did not require admission. ${ }^{11}$ Another study showed that $58 \%$ of all patients attended to by EMS required no prehospital clinical intervention (only transport) and concluded that EMS systems are unable to meet the demand as a result of such responses. ${ }^{12}$

Accuracy in dispatching resources according to triage is a key issue to bridge the gap between patient medical needs and EMS resources dispatched. ${ }^{13}$ This extends to existing dispatching protocols to be efficient and accurate in categorizing and triaging patients. International research all indicate a problem with overtriage resulting in the unnecessary response and transport of the low-acuity patient to hospital. ${ }^{13-15}$ Over-triage internationally ranges from $23 \%$ to as much as $78 \%$. Over-triage in South Africa has been reported to be as high as approximately $94 \%,{ }^{12}$ which is staggering in comparison to the rest of the world.

A popular system used in dispatching of EMS is the Medical Priority Dispatch System (MPDS). This dispatching system has reduced EMS response to calls of low acuity, however still has some problems in over-triage. ${ }^{16,17}$ Major aspects to be considered with this system are the cost, which is in excess of $\$ 10 \mathrm{M},{ }^{18}$ and the lack of validation of this system in a low- and middle-income country's (LMIC's) EMS environment, like South Africa. With eleven official languages, the diverse language profile of South Africa is another contributor to the accuracy of dispatch. This is particularly pronounced during emergency call taking, as the telephonic words and descriptors of the caller really provide the only clues for Emergency Medical Dispatch (EMD) staff to recognize and accurately assign acuity of patients. ${ }^{19}$

Research in the appropriate use of EMS resources has tried to define greater efficiencies in protocols used for the dispatching of resources, however this has been difficult. Organizations and countries operate independently and protocols coupled with outcome criteria for each EMS service vary, therefore studies have been difficult to evaluate. ${ }^{5}$ However, a consistent fact is that if the dispatch protocol is more accurate, this will reduce the amount of unnecessary resources being dispatched to lower acuity calls. Problems associated with low acuity are compounded by the fact that there is no clear evidence-based criteria to the concept of "low acuity in EMS," ${ }^{20}$ and those available are vague and vary subjectively between health care providers. ${ }^{21}$

The aim of this study is to develop consensus-based descriptors for low acuity in EMS within South Africa.

\section{Methods}

Design

A Modified Delphi study was conducted to obtain a set of consensus-based descriptors of low-acuity patients in EMS through experts in the field of prehospital and emergency medicine. These experts are well-experienced in EMS $^{22}$ and from various geographical locations around the country.

\section{Setting}

Emergency Medical Services in South Africa service patients through public and private systems. The public system is funded by the Department of Health, while private EMS service patients through reimbursement models such as medical insurance. In South Africa, $82 \%$ of the population does not have medical insurance and therefore relies on the public health care system. From an EMS perspective, there should be approximately 5,700 ambulances $(1 / 10,000 \text { population })^{23}$ in South Africa, but there are less than 2,000 ambulances in operation. ${ }^{10}$

\section{Sample and Sampling}

Participants recruited for this study were professional medical providers with tertiary qualifications within prehospital and emergency medicine. Participants were also required to be registered with the relevant professional regulating body in South Africa. Further, only participants with a minimum of two years operational experience within South Africa were eligible for participation.

The initial group of participants were nominated by the researcher via purposive sampling to ensure quality of the candidates. ${ }^{24,25}$ Participants were then provided the opportunity to nominate more candidates for the study through snowball sampling. A list of interested candidates with contact details was gathered and invited to participate in the study. ${ }^{22}$ These participants were evaluated by the researcher prior to invitation and were excluded if minimum recruitment criteria were not attained. The online survey tool Lime Survey (LimeSurvey GmbH; Hamburg, Germany) was used to conduct the study.

\section{Procedure}

This survey was conducted in three rounds. All participants were requested to provide informed consent for their participation.

The first round was developed through information gathered from literature available. ${ }^{16,17}$ Participants were asked for their agreement on three initial binary questions that should not be considered as low acuity (respiratory distress, unconsciousness, and severe hemorrhage). The balance of questions posed to the expert panel were open-ended questions under groups of potential emergency response categories as identified in the literature. Participants were asked to provide their expert opinion on criteria that could denote a patient as low acuity under each of the emergency response categories presented to them.

After the first round, open-ended questions within each emergency response category resulted in descriptors of low acuity being developed. Following content analysis, these descriptors were presented to the panel in round two through binary outcomes of "agree" or "disagree." Participants were also provided free-text 


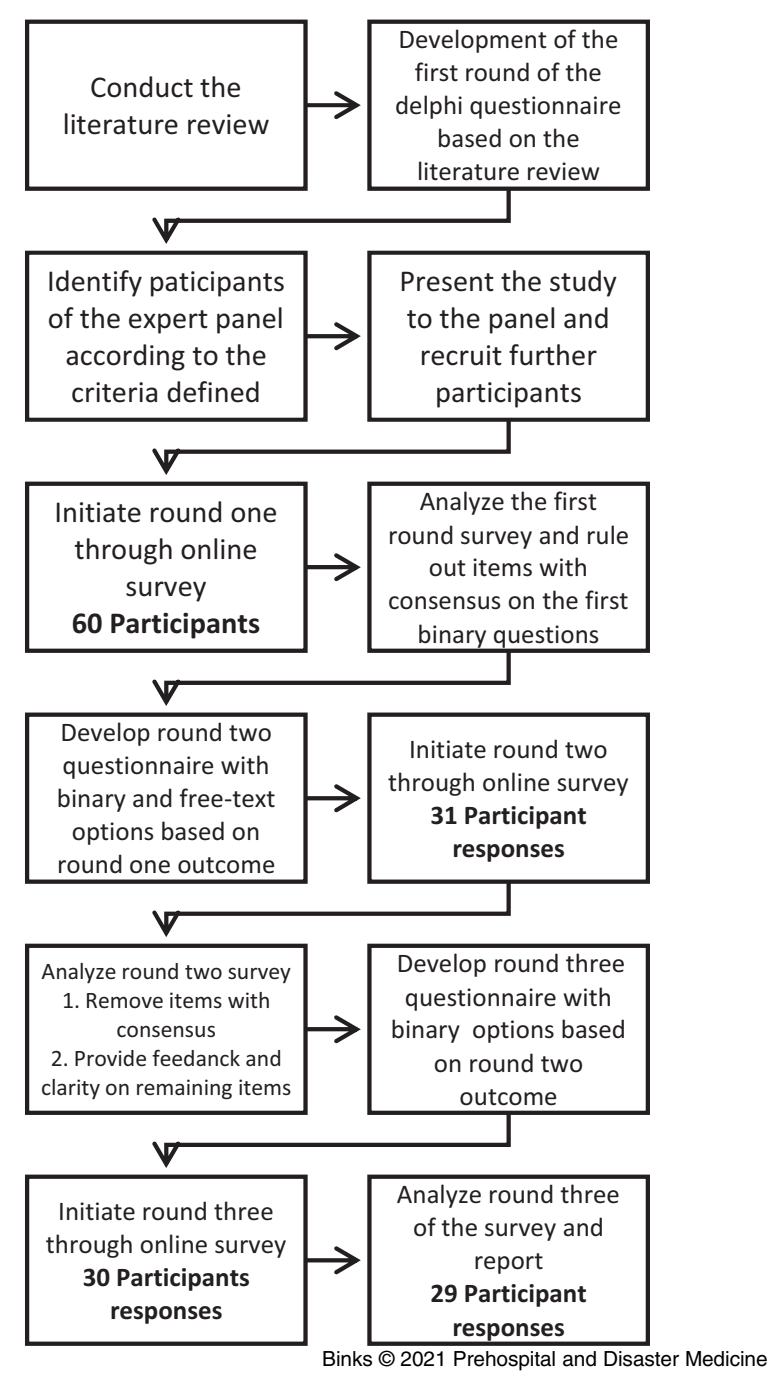

Figure 1. Process Flow of Delphi Study.

options to justify their responses. These comments were condensed and subjected to content analysis and presented to participants in round three which offered clarification and information for further consideration. Round three of the survey had binary outcomes only with no provision for free text in order to finalize consensus. Figure 1 depicts the process used.

Consensus for each descriptor was obtained when $75 \%$ of the participants either agreed or disagreed to the binary question. Consensus obtained on a particular descriptor would result in it either being excluded or included in the final list and was not presented to the participants in a subsequent round.

This study was approved by the Human Research Ethics Committee (HREC) of the University of Cape Town (Cape Town, South Africa; HREC Ref 491/2019). The results are presented in accordance with the proposed Conducting and REporting DElphi Studies (CREDES) checklist. ${ }^{26}$

\section{Results}

Invitations were sent to 60 candidates, 31 of whom completed round one of the survey providing a response rate of $52 \%$. In round two, 30 responses were received with an attrition rate of three percent. A further attrition rate of three percent was noted with 29 respondents in round three, providing a total response rate of

\begin{tabular}{|l|c|c|}
\hline Participants & $\mathbf{n}$ & $\%$ \\
\hline $\begin{array}{l}\text { Advanced Life } \\
\text { Support Practitioners }\end{array}$ & 26 & 90 \\
\hline Operations & 15 & 52 \\
\hline Management & 11 & 38 \\
\hline Doctors & 3 & 10 \\
\hline Experience & $\mathbf{n}$ & $\%$ \\
\hline 2 to 5 Years & 1 & 3 \\
\hline 6 to 10 Years & 6 & 21 \\
\hline$>11$ years & 22 & 76 \\
\hline
\end{tabular}

Table 1. Demographics of Participants

\begin{tabular}{|l|c|c|}
\hline Round 1 & $\mathbf{n}$ & \% \\
\hline $\begin{array}{l}\text { Consensus on } \\
\text { Questions }\end{array}$ & $3 / 3$ & 100 \\
\hline Round 2 & $\mathbf{n}$ & $\%$ \\
\hline $\begin{array}{l}\text { Consensus on } \\
\text { Questions }\end{array}$ & $67 / 152$ & 44 \\
\hline Round 3 & $\mathbf{n}$ & $\%$ \\
\hline $\begin{array}{l}\text { Consensus on } \\
\text { Questions }\end{array}$ & $46 / 85$ & 54 \\
\hline $\begin{array}{l}\text { Non-Consensus on } \\
\text { Questions }\end{array}$ & $10 / 155$ & 6 \\
\hline Exclusions & $29 / 155$ & 19 \\
\hline
\end{tabular}

Table 2. Summary of Consensus

94\% for the study. Candidates that completed all three rounds of the survey $(n=29)$ were either Advanced Life Support practitioners in EMS $(n=26)$ or a medical doctor employed in the field of prehospital or emergency medicine $(n=3)$. Demographics of the participants that completed all three rounds in the Delphi are presented in Table 1.

Table 2 provides a summary of the levels of consensus achieved through each round of the survey. Consensus was achieved in round one on all three binary disqualifier questions posed to participants, which were then excluded in the following rounds. Round one also yielded $(n=152)$ descriptors for all emergency response categories provided, which were then posed to participants in round two for consensus and opinion.

Round two of the survey achieved $44 \%(n=67)$ consensus on all binary questions, which were then excluded in round three. For the remaining questions in round two, the free text was analyzed, which resulted in questions being excluded $(n=29)$ and questions being clarified $(n=85)$ which were then asked again in round three. The final round achieved $54 \%(n=46)$ consensus on all questions, which resulted in an overall $75 \%$ consensus received on all binary questions through all three rounds.

Table 3 presents the descriptors that reached consensus on conclusion of the Delphi survey and therefore provide criteria for low acuity within each emergency response category.

\section{Discussion}

The aim of this study was to develop descriptors for low-acuity emergency calls for the South African context. "Low acuity" is a 


\begin{tabular}{|c|c|c|}
\hline Fundamental Descriptors & $\mathbf{n}$ & $\%$ \\
\hline No loss of consciousness & $27 / 31$ & 87 \\
\hline No form of respiratory distress & $28 / 31$ & 90 \\
\hline No signs of severe hemorrhage & $31 / 31$ & 100 \\
\hline Abdominal Pain & $\mathbf{n}$ & $\%$ \\
\hline $\begin{array}{l}\text { Abdominal pain in female patients of child-bearing age is considered low acuity if: } \\
\text { 1. Last menstrual period is obtained } \\
\text { 2. There is no history of undetected pregnancy }\end{array}$ & $23 / 29$ & 79 \\
\hline Abdominal pain is not related to trauma & $25 / 29$ & 86 \\
\hline No bleeding from any orifice & $26 / 29$ & 90 \\
\hline No associated fever with abdominal pain & $26 / 29$ & 90 \\
\hline Pre-existing condition with unchanged chronic pain & $27 / 30$ & 90 \\
\hline No signs of guarding to the abdomen & $26 / 30$ & 87 \\
\hline Patient is ambulant & $23 / 30$ & 77 \\
\hline Allergic Reactions & $\mathbf{n}$ & $\%$ \\
\hline Limited to skin reactions and topical symptoms only & $27 / 30$ & 90 \\
\hline Only one body system is affected, excluding respiratory/cardiovascular systems & $23 / 30$ & 77 \\
\hline No medical history of anaphylaxis or severe reactions & $24 / 29$ & 83 \\
\hline Animal Bites & $\mathbf{n}$ & $\%$ \\
\hline Minimal trauma or bleeding to the affected area & $27 / 29$ & 93 \\
\hline Animal bite is from a domestic pet that is up to date with its vaccination record & $27 / 29$ & 93 \\
\hline No requirement for any anti toxins & $23 / 30$ & 77 \\
\hline Patient is bitten by a known vaccinated animal & $24 / 29$ & 83 \\
\hline The animal has traceable medical records & $28 / 29$ & 97 \\
\hline Minimal injury to limb/extremity rather than face, neck, or head with no severe hemorrhage & $28 / 29$ & 97 \\
\hline Patient is an adult & $24 / 29$ & 83 \\
\hline Assault & $\mathbf{n}$ & $\%$ \\
\hline No central nervous system compromise & $26 / 30$ & 87 \\
\hline Minimal force on the patient is established & $23 / 30$ & 77 \\
\hline Superficial injury sustained & $28 / 30$ & 93 \\
\hline No significant pain felt by the patient & $23 / 30$ & 77 \\
\hline No penetrating trauma to head, neck, chest, and abdomen & $29 / 30$ & 97 \\
\hline Minimal trauma to extremities with no bleeding, deformity, fractures, or CNS compromise & $30 / 30$ & 100 \\
\hline Back Pain & $\mathbf{n}$ & $\%$ \\
\hline The patient is ambulant & $24 / 30$ & 80 \\
\hline Pain is bearable & $24 / 30$ & 80 \\
\hline No history of trauma experienced in the last 14 days & $25 / 29$ & 86 \\
\hline If related to trauma, pain must be bearable with no peripheral neurological compromise & $23 / 30$ & 77 \\
\hline $\begin{array}{l}\text { Back pain is not associated with other body systems such as cardiovascular, respiratory } \\
\text { systems, and urinary systems problems }\end{array}$ & $25 / 30$ & 83 \\
\hline There is no peripheral neurological compromise at all & $28 / 30$ & 93 \\
\hline Back pain is chronic in nature & $24 / 30$ & 80. \\
\hline No signs of infection noted & $25 / 30$ & 83 \\
\hline Back pain is related to minor muscle injury & $28 / 30$ & 93 \\
\hline Non-progressive back pain older than 24 hours & $29 / 29$ & 100 \\
\hline Burns & $\mathbf{n}$ & $\%$ \\
\hline The burn is superficial only & $28 / 30$ & 93 \\
\hline The superficial burn must be less than $10 \%$ BSA and exclude the head, neck, and genitalia & $22 / 29$ & 76 \\
\hline Chemical burns should not be considered low acuity & $24 / 30$ & 80 \\
\hline Electrical burns should not be considered low acuity & $26 / 30$ & 87 \\
\hline
\end{tabular}

Table 3. Descriptor Consensus for Low Acuity Calls in South Africa 


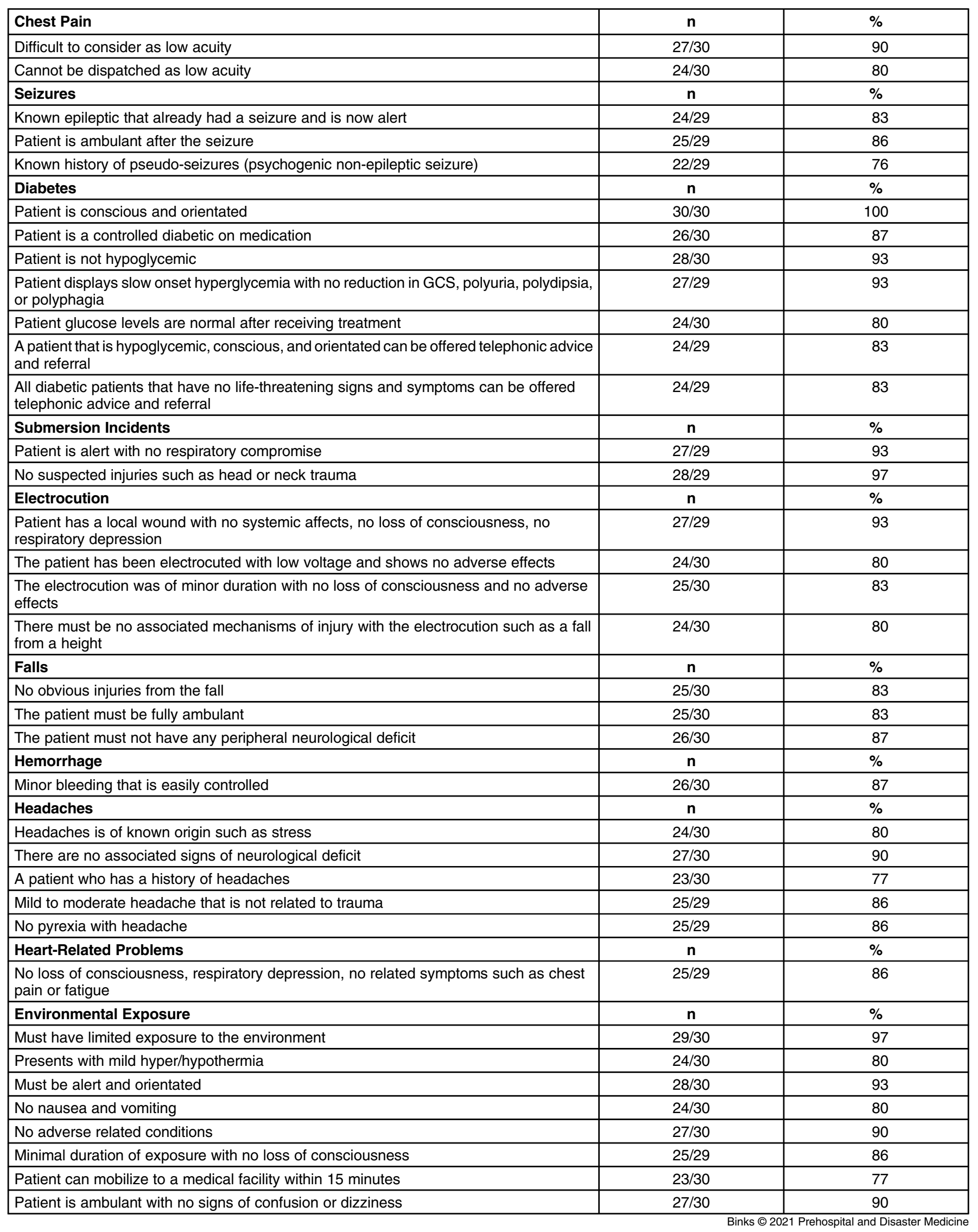

Table 3. Descriptor Consensus for Low Acuity Calls in South Africa 


\begin{tabular}{|c|c|c|}
\hline Overdose & $\mathbf{n}$ & $\%$ \\
\hline $\begin{array}{l}\text { Patient has taken medication more than } 24 \text { hours ago and shows no signs of acute } \\
\text { deterioration }\end{array}$ & $24 / 29$ & 83 \\
\hline Gynecology & $\mathbf{n}$ & $\%$ \\
\hline Usual regular menses with normal/slightly abnormal discharge & $28 / 30$ & 93 \\
\hline Patients that have menstruation cramps are low acuity & $26 / 30$ & 87 \\
\hline Abnormally high discharge during menstruation: patient to be referred & $25 / 29$ & 86 \\
\hline No potential pregnancy with abdominal pain & $24 / 30$ & 80 \\
\hline Pelvic inflammatory diseases with no immediate life-threatening signs and symptoms & $27 / 29$ & 93 \\
\hline Obstetrics & $\mathbf{n}$ & $\%$ \\
\hline Patient appointments or booked transports are low acuity & $25 / 30$ & 83 \\
\hline $\begin{array}{l}\text { A patient that is experiencing transient contractions with a good antenatal history and is } \\
\text { ambulant }\end{array}$ & $26 / 29$ & 90 \\
\hline Patient is not violent or aggressive & $27 / 30$ & 90 \\
\hline Patient is not a danger to themselves or anyone around them & $28 / 30$ & 93 \\
\hline Patient will not require physical or chemical restraint & $25 / 29$ & 86 \\
\hline Patient has a mild mood and anxiety disorder & $23 / 30$ & 77 \\
\hline No suicidal intentions & $26 / 30$ & 87 \\
\hline Penetrating Trauma & $\mathbf{n}$ & $\%$ \\
\hline Limb involvement with minor controlled bleeding and no loss of limb movement & $29 / 29$ & 100 \\
\hline No peripheral neurological compromise & $23 / 30$ & 77 \\
\hline Blunt Trauma & $\mathbf{n}$ & $\%$ \\
\hline Minimal or no pain to the patient following blunt trauma & $26 / 30$ & 87 \\
\hline Low speed RTA and patient has self-extricated & $27 / 29$ & 93 \\
\hline The patient must be ambulant & $28 / 29$ & 97 \\
\hline Patient must have been restrained in the vehicle & $26 / 29$ & 90 \\
\hline
\end{tabular}

Table 3. Descriptor Consensus for Low Acuity Calls in South Africa

Abbreviations: BSA, body surface area; CNS, central nervous system; GCS, Glasgow Coma Scale; RTA, road traffic accident.

term that categorizes patients subjectively and is vague in its definition ${ }^{21}$ making it difficult to apply operationally. Emergency calls in the EMD are also over-triaged as there are concerns that patients may die or staff may be subject to medicolegal litigation for incorrect dispatch. ${ }^{27}$

Determining which patients require an emergency response is a challenge and often results in disagreements between EMD staff and EMS operational staff. ${ }^{27} \mathrm{New}$ and safe dispatching protocols in the EMD are required for the reduction in unnecessary responses and transport of patients. ${ }^{27}$ Their development may be guided through the implementation of algorithms based on the criteria for low-acuity denotation identified in this study. However, the complexity of developing these algorithms is exemplified in these results as each emergency response category had (mostly) unique consensus-based criteria that should be applied.
The panel identified three universal criteria that disqualified cases from being deemed as low acuity. The panel felt that instances where loss of consciousness, respiratory distress, or severe hemorrhage are reported cannot be considered as low acuity. Respiratory distress is of particular interest as this was highlighted as one of the emergency response categories with a high risk for over-triage, but was also ranked as the third highest emergency category in the Western Cape, South Africa. ${ }^{27}$ Respiratory pathologies are ranked in the top ten causes of death in South Africa ${ }^{28,29}$ and were also shown to have a reduced discharge rate from the ED in a similar study conducted in Gauteng, South Africa. ${ }^{9}$ Comments received from the expert panel indicated that respiratory complaints are difficult to denote as low acuity telephonically because such a decision would be entirely dependent on an unknown caller's ability to assess a patient in respiratory distress. The risk in dispatching an emergency case with 
a patient in respiratory distress is evident in this study and therefore recommend that these cases be distinguished as high acuity with the development of an algorithm to enhance these dispatches.

The most prevalent cause of death in South Africa after HIV is cardiovascular disease, which has shown a remarkable increase from 2007 to $2017 .{ }^{28}$ The expert panel in this study felt very strongly that patients exhibiting chest pain cannot be categorized as low acuity. Statistics of call-outs from the EMD in the Western Cape for chest pain are slightly above two percent of the total emergency calls received, however all staff indicated that these emergency response categories carried a high risk of over-triage. ${ }^{27}$ The potential of overtriage for these chest pain patients are evident as common symptoms of a heart attack can be linked to other forms of pathologies, such as respiratory problems, heartburn, or even anxiety attacks. ${ }^{30}$

In the case then of respiratory distress and chest pain, it is perhaps not which calls to denote as low acuity, but instead to accept a priority response from the outset and develop algorithms that may instead distinguish those patients with higher risk from the others. ${ }^{31}$ This may ensure that those prehospital providers with the requisite scope of practice and equipment to manage patients with asthma and myocardial infarction be dispatched preferentially. These algorithms will be heavily reliant on the caller's description of the patient. ${ }^{30}$

The highest discharge rate for medical patients in Gauteng brought in by ambulance was among those with gastrointestinal complaints (36\%). ${ }^{9}$ Gastrointestinal emergencies also constitute almost $11 \%$ of emergency responses in the Western Cape. ${ }^{27}$ The high rates of call-outs, together with discharge rates, indicate that these patients may not have warranted transport to hospital but may have benefitted from other medical assistance such as referral to primary health care facilities or telephonic advice. Consideration must be provided for confounding factors in these cases such as the prevalence of gastrointestinal complaints found in patients with sepsis; a South African study found that $40 \%$ of patients with the prehospital diagnosis of sepsis were described as having gastrointestinal symptoms at initial emergency telephone call. ${ }^{19}$ The consequent descriptors from the expert panel may provide distinguishing criteria for low-acuity cases in reducing the high call-out rates and therefore contribute to the development of algorithms in reducing unnecessary dispatch of abdominal cases.

Obstetric emergencies in Africa have been the second highest call-out volume for $\mathrm{EMS}^{32}$ and make up six percent of call-outs in the Western Cape. ${ }^{27}$ Although delays in these responses contribute to maternal mortality through obstetric complications, ${ }^{33}$ implementation of any dispatch protocol in this respect requires meticulous consideration in order to mitigate maternal deaths, which are known to be as high as $84 \%$ in LMICs. ${ }^{34}$

Road traffic accidents (RTAs; 47\%) and patient assaults (37\%) accounted for $83 \%$ of trauma-related discharges brought in by ambulance. ${ }^{9}$ These statistics are significant, especially if there exist mechanisms to redirect these patients from the ED, providing efficiency to the health care system. Not dispatching emergency vehicles to RTAs received via telephone may be a challenge as injuries at the time of the call may not be known; however, the descriptors may provide value in supporting other systems like on-scene discharge to limit the unnecessary transportation of patients to the ED. Many of the patients in these cases with minor medical requirements, like alleviation of pain or tetanus toxoid injections, may be transported or preferably referred to primary care clinics after evaluation by emergency medical staff. ${ }^{34}$

The development of algorithms using these descriptors in each call-out category may not only terminate responses to calls of low acuity, but may also provide distinguishing criteria between highacuity and low-acuity dispatch models and contribute to alternative dispatch systems that can influence policy of response to calls. This may be in the form of a time critical response approach to some calls where lights and sirens are required, or other cases where an acceptable time frame for response may be determined through evidence-based decisions. The implementation of algorithms in dispatching emergency resources to calls will also support other EMS systems such as on-scene discharge or telephonic advice systems. The challenge of receiving emergency calls in the EMD in a multilingual country with varying levels of literacy and education may also be alleviated through protocol-driven algorithms which can be informed through the low-acuity descriptors of this study.

\section{Limitations}

The results and recommendations of this study are based on expert opinion, which represents a low level of evidence-based medicine. For this reason, further development of these criteria is recommended as well as future research to refine and test these results.

Participants for this study were all South African registered emergency care practitioners and medical doctors to ensure that descriptors were contextual to the setting. This might limit the external validity of the study and results should be applied to other settings with caution.

\section{Conclusion}

The results of this study have provided a set of descriptors in order to define low acuity within the EMS environment of South Africa. A significant level of responsibility lies with the EMD center in reducing the dispatch of resources to low-acuity calls. These descriptors may be developed into algorithms that will affect an appropriate response to emergency calls. Further research is required in validating the application of these definitions in the EMS environment.

\footnotetext{
References

1. Dami F, Golay C, Pasquier M, Fuchs V, Carron PN, Hugli O. Prehospital triage accuracy in a criteria-based dispatch center. BMC Emerg Med. 2015;15(1):32.

2. Eastwood K, Morgans A, Smith K, Hodgkinson A, Becker G, Stoelwinder J. A novel approach for managing the growing demand for ambulance services by low-acuity patients. Aust Heal Rev. 2016;40(4):378.

3. Sofianopoulos S, Williams B, Archer F, Thompson B. The exploration of physical fatigue, sleep and depression in paramedics: a pilot study. J Emerg Prim Health Care. 2011;9(1):1-33.

4. Bayati M, Kwasnick S, Luo D, Plambeck EL. Low-Acuity Patients Delay HighAcuity Patients in an Emergency Department. SSRN. 2018.
}

5. Munjal KG, Belachew A, Tan N, et al. 136-Retrospective application of a low acuity Emergency Medical Services triage protocol to identify patients appropriate for urgent care. Ann Emerg Med. 2015;66(4):S48.

6. Alpert A, Morganti KG, Margolis GS, Wasserman J, Kellermann AL. Giving EMS flexibility in transporting low-acuity patients could generate substantial Medicare savings. Health Aff. 2013;32(12):2142-2148.

7. Dale J, Williams S, Foster T, et al. Safety of telephone consultation for 'non-serious' emergency ambulance service patients. Qual Saf Heal Care. 2004;13(5):363-373.

8. Cameron PA, Gabbe BJ, Smith K, Mitra B. Triaging the right patient to the right place in the shortest time. Br J Anaesth. 2014;113(2):226-233. 
9. Goldstein LN, Sibanyoni S, Vincent-Lambert C. On-scene discharge by emergency care practitioners-a viable option for South Africa? South African J Prehosp Emerg Care. 2020;1(1):3-8.

10. Graphic of the day: where are South Africa's ambulances? Bhekisisa. https://bhekisisa. org/article/2018-12-19-00-graphic-of-the-day-where-are-south-africas-ambulances/ . Accessed June 15, 2020.

11. Hodkinson PW, Wallis LA. Cross-sectional survey of patients presenting to a South African urban emergency center. Emerg Med J. 2009;26(9):635-640.

12. Newton P. An evaluation of the appropriateness of emergency medical service (EMS) responses in the eThekwini health district of KwaZulu-Natal. 2014. http://hdl.handle. net/10321/1105. Accessed June 15, 2020.

13. Stassen W, Larsson E, Wood C, Kurland L. Telephonic description of sepsis among callers to an emergency dispatch center in South Africa. African J Emerg Med. 2020;10(2):64-67.

14. Khorram-Manesh A, Montán KL, Hedelin A, Kihlgren M, Örtenwall P. Prehospital triage, discrepancy in priority-setting between emergency medical dispatch center and ambulance crews. Eur J Trauma Emerg Surg. 2011;37(1):73-78.

15. Feldman MJ, Verbeek PR, Lyons DG, Chad SJ, Craig AM, Schwartz B. Comparison of the Medical Priority Dispatch System to an out-of-hospital patient acuity score. Acad Emerg Med. 2006;13(9):954-960.

16. Sporer KA, Craig AM, Johnson NJ, Yeh CC. Does emergency medical dispatch priority predict Delphi process-derived levels of prehospital intervention? Prehosp Disaster Med. 2010;25(4):309-317.

17. Clawson J, Olola C, Heward A, Patterson B, Scott G. Ability of the medical priority dispatch system protocol to predict the acuity of 'unknown problem' dispatch response levels. Prehosp Emerg Care. 2008;12(3):290-296.

18. Recommendations from the Provincial Municipal Land Ambulance Dispatch Working Group Submission to the Minister of Health and Long-Term Care. 2015.

19. Mann NC, Schmidt TA, Cone DC. "Defining Research Criteria to Characterize Medical Necessity in Emergency Medical Services: A Consensus among Experts at the Neely Conference." In: Prehospital Emergency Care. No longer published by Elsevier; 2004:138-153.

20. Cone DC, Galante N, MacMillan DS. Can emergency medical dispatch systems safely reduce first-responder call volume? Prehosp Emerg Care. 2008;12(4):479-485.
21. Hasson F, Keeney S, Mckenna H. Research guidelines for the Delphi survey technique. Artic J Adv Nurs. 2000;32(4):1008-1015.

22. Annual Reports - KZN Department of Health. http://www.kznhealth.gov.za/reports. htm. Accessed July 9, 2020.

23. Ludwig B. Predicting the future: Have you considered using the Delphi methodology? J Ext. 1997;35(5):5TOT2.

24. Oh K. Forecasting through hierarchical Delphi. 1974. https://etd.ohiolink.edu/ apexprod/rws_etd/send_file/send?accession=osu148698532753885\&disposition=inline. Accessed July 9, 2020.

25. Jünger S, Payne SA, Brine J, Radbruch L, Brearley SG. Guidance on Conducting and REporting DElphi Studies (CREDES) in palliative care: recommendations based on a methodological systematic review. Palliat Med. 2017;31(8):684-706.

26. Alshehri MF, Pigoga JL, Wallis LA. A mixed methods investigation of emergency communications center triage in the Government Emergency Medical Services System, Cape Town, South Africa. African J Emerg Med. 2020;10(Suppl 1):s12-s17.

27. South Africa - Institute for Health Metrics and Evaluation. University of Washington. http://www.healthdata.org/south-africa. Accessed July 12, 2020.

28. Statistics South Africa. People - Statistics South Africa. 2014: People-Labour market. www.statssa.gov. Accessed July 12, 2020.

29. Health Advisor. Know how to interpret different types of chest pain or discomfort: it's not always easy, but distinguishing between heartburn and a heart attack may save you some stress and save your life. Cleveland Clinic. 2017;20(2):7-8.

30. Buma C, Saunders C, Watermeyer J, Stassen W. An analysis of the descriptors of acute myocardial infarction used by South Africans when calling for an ambulance from a private emergency call center. African J Emerg Med. 2020;10(4):203-208.

31. Mould-Millman NK, Dixon JM, Sefa N, et al. The state of Emergency Medical Services (EMS) systems in Africa. Prehosp Disaster Med. 2017;32(3):273-283.

32. Thaddeus S, Maine D. Too far to walk: maternal mortality in context. Soc Sci Med. 1994;38(8):1091-1110.

33. Pacagnella RC, Cecatti JG, Parpinelli MA, et al. Delays in receiving obstetric care and poor maternal outcomes: results from a national multicenter cross-sectional study. BMC Pregnancy Childbirth. 2014;14(1):1-15.

34. Gilboy N, Tanabe T, Travers D RA, Gilboy N Travers D, Rosenau A TP. Emergency Severity Index (ESI): A Triage Tool for Emergency Department Care, Version 4. Rockville, Maryland USA: Agency for Healthcare Research and Quality; 2012:69. 